\title{
$\alpha$-Ketoacid dehydrogenase complexes and respiratory fuel utilisation in diabetes
}

\author{
P.J.Randle \\ Nuffied Department of Clinical Biochemistry, University of Oxford, John Radcliffe Hospital, Oxford, UK
}

\begin{abstract}
Summary. Activity of the pyruvate dehydrogenase complex determines the rate of glucose oxidation in animals including man. The complex is regulated by reversible phosphorylation, phosphorylation resulting in inactivation. Activity is therefore dependent upon the activities of pyruvate dehydrogenase kinase and phosphatase. Activity of the complex is reduced in diabetes and starvation as a result of insulin deficiency. The mechanism involves activation of pyruvate dehydrogenase kinase by short-term effects of products of fatty acid oxidation and by longer term effects involving specific protein synthesis; in hepatocytes the signals may include lipid fuels and glucagon. Activity of the branched chain ketoacid dehydrogenase complex determines the rate of degradation of branched chain aminoacids which is adjusted according to dietary sup-
\end{abstract}

ply. The complex is regulated by reversible phosphorylation, phosphorylation being inactivating. In liver and kidney, but not in muscles a protein activator (free El component) may reactivate phosphorylated complex without dephosphorylation and facilitate hepatic oxidation of branched chain ketoacids. Metabolic adjustments induced by diet and diabetes include loss of activator protein, loss of total complex activity in liver but not muscles, and enhanced inactivation by phosphorylation in liver.

Key words: Pyruvate dehydrogenase complex, branched chain ketoacid dehydrogenase complex, diabetes, starvation, low protein diets, activator protein.
The first Minkowski lecture in 1966 was on "Carbohydrate metabolism and lipid storage and breakdown" [1]. At that time I was interested in the effects of lipid oxidation on carbohydrate metabolism; and also in the regulation of insulin secretion, especially the mechanisms mediating glucose stimulated insulin secretion. My current interest in ketoacid dehydrogenase complexes in relation to respiratory fuels in diabetes had its origins in the subjects of the first Minkowski lecture. I gave up active research into the regulation of insulin secretion in 1973. Dr. S.J.H. Ashcroft, who continued this line of research, became the fourteenth Minkowski lecturer in 1979 [2]. His studies through the 1970s provided compelling evidence for the idea that insulin secretory responses to sugars involve $\beta$-cell metabolism of the sugars [2]. The recent discovery of a $\beta$-cell membrane ion channel sensitive to glucose metabolism by F.M. Ashcroft, D.E. Harrison and S.J.H. Ashcroft [3] may provide the breakthrough needed to discover the underlying mechanism.

The concept of a 'glucose fatty acid cycle' which was the subject of the first Minkowski lecture has survived and been extended by others to incorporate gluconeogenesis [4]. There have been doubts about its applicability to skeletal muscle, but these have been largely dispelled by more careful studies [5]. The major change has been in details of mechanism, occasioned mainly by the discovery of reversible phosphorylation in the pyruvate dehydrogenase complex by Dr L.J.Reed and his colleagues [6]. It was this discovery which reawakened my own interest in the pyruvate dehydrogenase complex and led ultimately to my interest in the branched chain complex.

\section{Ketoacid dehydrogenase complexes}

\section{Chemistry and substrates}

The $\alpha$-ketoacid dehydrogenase complexes catalyse reactions of the general type shown in equation 1 (TPP is thiamin pyrophosphate).

$$
\begin{aligned}
& \text { R.CO.COOH + NAD + CoA } \stackrel{\text { TPP }}{\longrightarrow} \text { R.CO.CoA }+ \\
& \mathrm{NADH}_{2}+\mathrm{CO}_{2}
\end{aligned}
$$

There are three such complexes (all mitochondrial) the pyruvate dehydrogenase (PDH) complex; the $\alpha$-ketoglutarate dehydrogenase complex and the branched chain $\alpha$-ketoacid dehydrogenase (BCKDH) complex. The BCKDH complex oxidises $\alpha$-ketoacids corresponding to the amino acids leucine, isoleucine and valine (referred to as ketoleucine, ketoisoleucine and ketovaline). This paper is concerned with the PDH complex and the BCKDH complex.

The complexes contain multiple copies of three component enzymes. These are referred to as El (which 
decarboxylates the $\alpha$-ketoacid forming $\left.\mathrm{CO}_{2}\right) ; \mathrm{E} 2$ (which forms the acyl CoA product); and E3 (lipoyl dehydrogenase) which is common to all three complexes and which forms $\mathrm{NADH}_{2}$. The reactive components (acyl group and hydrogen) are shuttled between the component enzymes by a dithiol compound (lipoate) attached to a swinging arm on E2, Eq. (2-4). The core of the complex

R.CO.COOH + (SS.lip.)E2 $\rightarrow$ (R.CO.SSH.lip.) $\mathrm{E} 2+\mathrm{CO}_{2}$ (R.CO.SSH.lip.)E2 + CoASH $\leftrightharpoons$ R.CO.SCoA + (SHSH.lip.)E2 (SHSH.lip.)E2 + NAD $\leftrightharpoons$ (SS.lip.)E2 + $\mathrm{NADH}_{2}$

is formed by $\mathrm{E} 2$ (60 copies of a single subunit in PDH complex; probably 24 copies of a single subunit in $\mathrm{BCKDH}$ complex) and $\mathrm{E} 1$ and $\mathrm{E} 3$ are distributed on the surface of E2. The E1 component is a tetramer of two dissimilar subunits $\left(\alpha_{2} \mathrm{~B}_{2}\right)$ (PDH and BCKDH complexes). The complexes are located in the inner mitochondrial membrane and utilise only intramitochondrial substrates and coenzymes. The holocomplex reaction, Eq. (1), is essentially irreversible (equilibrium constant, approximately $10^{7}$ ). This is due to irreversibility of the reaction catalysed by E1, Eq. (2); the reactions catalysed by E2 and E3 are reversible, Equations (3) and (4). More detailed reviews of the biochemistry of these complexes are given in $[7,8]$.

\section{Biomedical significance}

The activity of the PDH complex determines the rate of glucose degradation in animals including man, because of the operation of the Cori cycle. Although glycolysis in irreversible and glycolytic flux (formation of pyruvate) exceeds pyruvate oxidation, glucose is resynthesised from the glycolytic products by gluconeogenesis in the liver (and to a lesser extent in kidney) (Fig.1). Glucose oxidation is decreased markedly in uncon-

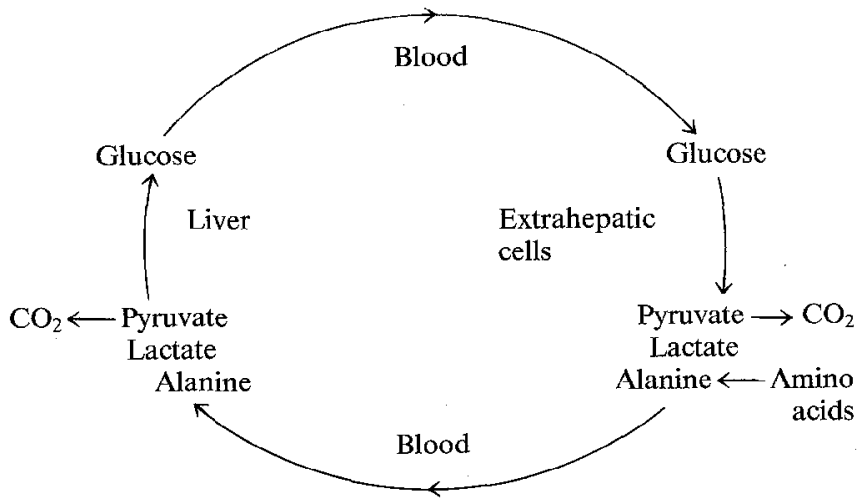

Fig. 1. Glucose recycling, utilisation and production trolled Type 1 (insulin-dependent) diabetes [9] and in many cases of uncontrolled Type 2 (non-insulin-dependent) diabetes. Glucose oxidation is also reduced markedly by starvation in man and other animals. Evidence is reviewed later to show that these effects of diabetes and starvation are mediated through inactivation of the PDH complex by phosphorylation. In muscles glycolytic flux is decreased when glucose oxidation is decreased and this reduction in glycolytic flux is also apparently dependent on inactivation of the PDH complex (see below). The PDH complex is also involved in biosynthesis of fatty acids from glucose. Regulation of its activity by insulin in rat adipose tissue is important in regulation of fatty acid biosynthesis in this tissue. Regulation of PDH complex by insulin in liver is uncertain and therefore the role of this regulation in fatty acid biosynthesis in man (predominantly hepatic) is also uncertain [10, 11].

Branched chain amino-acids are essential amino-acids and dietary requirement at nitrogen equilibrium is determined by the rate of their degradation (for pathways, Fig. 2). The rate of degradation is determined by the activity of the BCKDH complex which, above an irreducible minimum, is adjusted according to dietary supply. The activity of BCKDH complex is also a major factor determining circulating concentrations of branched chain amino- and ketoacids. This is important because excessive concentrations are toxic. Maple syrup urine disease is an inborn error of metabolism in which, as a result of mutation, the $\mathrm{K}_{\mathrm{m}}$ of $\mathrm{BCKDH}$ complex for branched chain ketoacids is increased approximately 100 -fold. As a consequence circulating concentrations of branched chain amino- and ketoacids are increased up to 70-fold. Brain damage ensues and affected infants, if untreated, rarely survive beyond the age of 2 years. Transamination of branched chain amino-acids is predominantly extrahepatic and branched chain ketoacids are released into the circulation and degraded in the liver. As will be seen later, tissue specific regulation of the activity of BCKDH complex may determine relative rates of hepatic and extrahepatic degradation of branched chain ketoacids.

Leucine (and ketoleucine) have important regulatory functions. They stimulate insulin secretion; and leucine may activate protein synthesis and inhibit protein degradation by direct action on tissues. In starvation and in uncontrolled Type 1 diabetes in man circulating concentrations of branched chain amino-acids are increased specifically ( 2 to 3 -fold). This may be a feedback mechanism restraining rates of tissue protein loss and the increase in circulating concentrations of branched chain amino acids $[12,13]$.

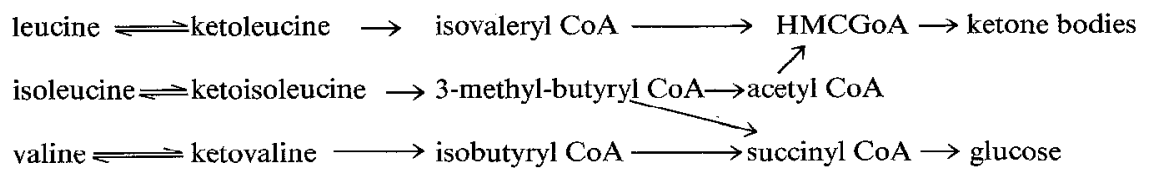

transaminases branched various

Fig. 2. Metabolism of branched chain amino acids 


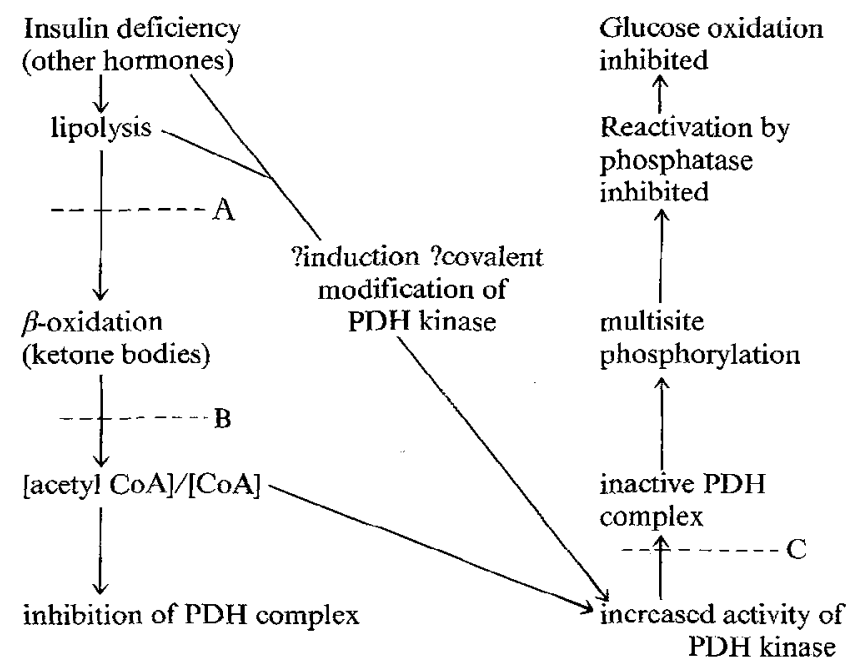

Fig. 3. Molecular mechanisms inactivating PDH complex and inhibiting glucose oxidation in starvation or diabetes. Glucose oxidation and percentage active $\mathrm{PDH}$ complex are increased in starved or diabetic animals by drugs which inthibit lipolysis (A) or $\beta$-oxidation of fatty acids (B) or PDK kinase (C)

\section{Regulation of the pyruvate dehydrogenase complex}

The PDH complex is inhibited by its end products such that activity is inversely correlated with mitochondrial concentration ratios of acetylCoA/CoA and $\mathrm{NADH}_{2} / \mathrm{NAD}$. These ratios are increased by oxidation of lipid fuels (fatty acids, ketone bodies) [1]. More importantly PDH complex is regulated by reversible phosphorylation. PDH kinase (an integral component of the complex) catalyses phosphorylation of a serine residue in an $\alpha$-subunit of E1 resulting in inactivation of PDH complex, Eq. (5). Two further phosphorylations occur with no further change in activity, Eq. (6). Dephosphorylation and reactivation are effected by $\mathrm{PDH}$

$\mathrm{PDH}$ complex $(\mathrm{E} 1 \alpha)+\mathrm{ATP} \rightarrow \mathrm{PDH}$ complex(E1 $\alpha \mathrm{P})+\mathrm{ADP}(5)$
$\mathrm{PDH}$ complex $(\mathrm{E} 1 \alpha \mathrm{P})+2 \mathrm{ATP} \rightarrow \mathrm{PDH}$ complex $\left(\mathrm{E} 1 \alpha \mathrm{P}_{3}\right)+2 \mathrm{ADP}$

$\mathrm{PDH}$ complex $\left(\mathrm{E} 1 \alpha \mathrm{P}_{\mathrm{n}}\right)+\mathrm{nH}_{2} \mathrm{O} \rightarrow \mathrm{PDH}$ complex $(\mathrm{E} 1 \alpha)+\mathrm{nP}_{\mathrm{i}}\left({ }^{\prime}\right)$

phosphatase, Eq. (7). The rate of reactivation by $\mathrm{PDH}$ phosphatase is decreased by multisite phosphorylations, Eq. (6) [7, 8, 14].

Activity of the complex in vivo depends upon the proportion in the active (dephosphorylated) form; total PDH complex (active plus inactive forms) remains unchanged. The kinase and phosphatase reactions operate simultaneously in vivo and the activity of PDH complex is therefore regulated by the relative activities of $\mathrm{PDH}$ kinase and phosphatase.

\section{Regulation of PDH kinase and phosphatase}

The PDH kinase reaction is regulated by mitochondrial concentration ratios of three metabolite pairs, ATP/ $\mathrm{ADP}$, acetyl $\mathrm{CoA} / \mathrm{CoA}$ and $\mathrm{NADH}_{2} / \mathrm{NAD}$; increasing concentration ratios activate $\mathrm{PDH}$ kinase. It is inhibited by its other substrates (pyruvate, TPP) and by haloge- nated carboxylates such as dichloroacetate. PDH phosphatase requires $\mathrm{Mg}^{2+}$ for activity and with $\mathrm{Mg}^{2+}$ is activated by $\mathrm{Ca}^{2+}$ over the physiological range $\left(10^{-8}-10^{-5} \mathrm{~mol} / 1\right)[7,8]$.

\section{Lipid fuels and muscle work}

In rat muscles, liver and kidney oxidation of lipid fuels decreases glucose oxidation and percentage active PDH complex. In heart muscle, for example, the decrease in active PDH complex is from approximately $30 \%$ to $7 \%[10,15]$. The mechanism involves activation of PDH kinase by an increase mitochondrial concentration ratio of acetyl $\mathrm{CoA} / \mathrm{CoA}$ and the effects of fatty acids are blocked by inhibitors of fatty acid oxidation (e.g. 2 -tetradecylglycidate) $[16,17]$. In muscles (cardiac or skeletal) work increases glucose oxidation and the percentage active complex. In heart muscle, for example, pressure development increases percentage active $\mathrm{PDH}$ complex from approximately $30 \%$ to $80 \%$. The mechanism involves activation of PDH phosphatase by increased mitochondrial $\mathrm{Ca}^{2+}$ (secondary to increased cytosolic $\mathrm{Ca}^{2+}$ ) and inhibition of PDH kinase by decreased mitochondrial concentration ratios of the metabolite pairs [18, 19].

\section{Mechanisms mediating effects of diabetes and starvation}

Induction of pancreatic diabetes (alloxan or streptozocin) or starvation $(48 \mathrm{~h})$ in rats markedly decreases glucose oxidation and percentage active PDH complex in muscles (heart or skeletal), liver, kidney and adipose tissue. The decrease is substantial; for example, in heart percentage active complex is decreased from approximately $30 \%$ to $1-2 \%$. The effects of diabetes are reversed by insulin in vivo (12-48 h) and those of starvation by refeeding (12-48 h). The effects of diabetes and starvation are not reversed by insulin plus glucose in vitro $[15,16]$ (i. e. they are not short-term effects of insulin deficiency). The effect of diabetes or starvation on percentage active PDH complex requires increased oxidation of lipid fuels; in heart or kidney, for example, their effects may be reversed with 2-tetradecyl glycidate.

A number of lines of evidence show that short-term effects of increased oxidation of lipid fuels is not solely responsible for the effects of pancreatic diabetes or starvation. The effects of diabetes and starvation on percentage active complex are more marked than those of oxidation of lipid fuels. The effect of pancreatic diabetes or starvation to decrease per cent active complex persists into mitochondria prepared from tissues and in the absence of lipid fuels. The activity of PDH kinase in extracts of mitochondria containing PDH complex and prepared from tissues of diabetic or starved rats is increased relative to controls (approximately 2.5 to 3 -fold). Recent studies have shown that the activity of PDH kinase in PDH complex purified from heart mitochondrial extracts of starved rats is increased 2.5-fold relative to controls. A second protein fraction, which may be separated from PDH complex by gel filtration 
or high speed centrifugation, increases activity of PDH kinase; the activity of this fraction is increased by diabetes or starvation. The increased activity of this fraction requires cytoplasmic protein synthesis (shown with inhibitors of protein synthesis, cycloheximide puromycin) $[16,20]$.

The model summarising mechanisms to explain effects of pancreatic diabetes or starvation is shown in Figure 3. In this model it is suggested that diabetes or starvation may result in increased synthesis of PDH kinase and an increased concentration of PDH kinase in the complex. Alternatively, PDH kinase is increased in activity (not concentration) by a stable mechanism, such as covalent modification. The protein fraction which increases activity of PDH kinase could either contain PDH kinase or an activator of PDH kinase. In principle this problem (i.e. the distinction between the two possibilities) is soluble but it is difficult in practice because of tight binding of PDH kinase in the complex and the small quantities of material available.

The factors (hormonal, metabolic) that may be responsible for the long-term adaptive change which increase PDH kinase activity is being studied by tissue culture of rat hepatocytes for periods up to $48 \mathrm{~h}$. Preliminary results suggest that PDH kinase activity in extracts of mitochondria from cultured hepatocytes is increased approximately 2.5 -fold by $21 \mathrm{~h}$ of exposure to a combination of glucagon and a fatty acid (e.g. octanoate) H.R. Fatania, T.C.Vary and P.J.Randle, unpublished observations). If these preliminary findings are substantiated by more detailed studies then the original formulation of the role of glucose fatty acid cycle will remain. It will, however, have been modified in detail to incorporate reversible phosphorylation in the PDH complex and longer term effects of fatty acids involving, presumably, regulation of specific protein synthesis.

\section{Interactions with glycolysis}

When oxidation of glucose (or pyruvate) and percentage active PDH complex are decreased in muscles by oxidation of lipid fuels or by diabetes glycolysis is inhibited at the level of phosphofructokinase [1]. This inhibition of glycolysis is reversed when inhibitors of PDH kinase increase glucose oxidation and percent active PDH complex. Inhibition of phosphofructokinase is known to be dependent upon accumulation of citrate [1] which in turn is dependent upon transamination between glutamate and pyruvate. Inactivation of PDH complex by phosphorylation is apparently necessary to sustain the pyruvate concentration required for citrate accumulation [10].

\section{Relevance to man and to human diabetes}

It is known that PDH complex from human tissues is regulated by reversible phosphorylation [21]. It is known also that glucose oxidation is inhibited in poorly controlled diabetes. The results of biochemical measurements in diabetic patients treated with dichloroace- tate are consistent with an action of the drug to increase glucose oxidation through inhibition of PDH kinase and a consequential increase in percent active PDH complex.

When details of the scheme shown in Figure 3 have been substantiated in animal models, it should be possible to ascertain whether they may have their counterpart in man. The effect of the long-term stable mechanism(s) which activate PDH kinase in diabetic animals is to set percentage active PDH complex at a lower level under all conditions. Thus although, for example, muscle work increases percentage PDH complex in diabetes, the level of activity achieved remains below that of the non-diabetic control. The mechanism(s) are likely to play a part in other metabolic complications of diabetes, e.g. the development of lactacidosis in association with diabetic ketoacidosis.

\section{Regulation of branched chain ketoacid dehydrogenase complex}

The BCKDH complex is also regulated by end product inhibition through mitochondrial concentration ratios of branched chain acyl $\mathrm{CoA} / \mathrm{CoA}$ and $\mathrm{NADH}_{2} / \mathrm{NAD}$. It is also regulated by reversible phosphorylation. A $\mathrm{BCKDH}$ kinase (an integral component of the complex) catalyses phosphorylation of two serine residues in the $\alpha$-subunit of E1 resulting in inactivation. A specific BCKDH phosphatase catalyses dephosphorylation and reactivation $[13,22]$. The equations are similar to those given for PDH complex, Eq. (5-7) except that only two serine residues are involved. Phosphorylated (inactive) $\mathrm{BCKDH}$ complex is also reactivated but without dephosphorylation by a protein (activator protein) which is present in liver and kidney mitochondria but which has not been detected in heart or skeletal muscle mitochondria. Activator protein has been purified extensively, and is apparently the free E1 component of the complex (or an isozyme of E1). In liver and kidney but not in muscles E1 is apparently synthesised in excess of requirment for holocomplex assembly and can bypass phosphorylated $\mathrm{E} 1$ in the complex) $[22,23]$.

\section{Regulation of $B C K D H$ kinase and phosphatase}

BCKDH kinase is inhibited by ketoacid substrates (mainly ketoleucine and ketoisoleucine), by TPP (thiamin pyrophosphate) and by ADP, acetoacetylCoA and malonyl CoA. Regulation of the phosphatase is currently unknown [22].

\section{Tissue specific regulation}

Liver and kidney, and liver and kidney mitochondria contain predominantly active complex whereas heart and skeletal muscle (or mitochondria therefrom) contain predominantly inactive complex. Interconversion of active and inactive forms is readily demonstrable in muscle mitochondria, less readily in kidney mitochon- 
Table 1. Activities of branched chain complex in rat liver, kidney, and heart: effects of diet and alloxan diabetes

\begin{tabular}{|c|c|c|c|c|c|c|}
\hline \multirow[t]{3}{*}{ Rat (diet) } & \multicolumn{6}{|c|}{ Branched chain complex (unit/g wet wt of tissue) in: } \\
\hline & \multicolumn{2}{|l|}{ Liver } & \multicolumn{2}{|l|}{ Kidney } & \multicolumn{2}{|l|}{ Heart } \\
\hline & Total & $\%$ active & Total & $\%$ active & Total & $\%$ active \\
\hline Normal (standard) & $0.82 \pm 0.06$ & $55 \pm 4.6$ & $0.77 \pm 0.07$ & $71 \pm 4.9$ & $0.57 \pm 0.04$ & $5.3 \pm 0.5$ \\
\hline Normal ( $80 \%$ casein) & $0.79 \pm 0.07$ & $30 \pm 1.5$ & $0.87 \pm 0.07$ & $47 \pm 3.8$ & $0.53 \pm 0.03$ & $8.9 \pm 1.8$ \\
\hline Normal ( $9 \%$ casein) & $0.32 \pm 0.04^{\mathrm{a}}$ & $14 \pm 1.0^{\mathrm{a}}$ & $0.77 \pm 0.04$ & $21 \pm 2.8^{\mathrm{a}}$ & $0.57 \pm 0.04$ & $10.9 \pm 1.9$ \\
\hline Normal $(0 \%$ casein $)$ & $0.25 \pm 0.05^{\mathrm{a}}$ & $29 \pm 3.3^{\mathrm{a}}$ & $0.66 \pm 0.03$ & $26 \pm 5.9^{\mathrm{a}}$ & $0.62 \pm 0.03$ & $6.0 \pm 2.2$ \\
\hline Normal (48 h starved) & $0.61 \pm 0.04^{\mathrm{a}}$ & $10 \pm 0.7^{\mathrm{a}}$ & $0.61 \pm 0.02$ & $32 \pm 2.6^{\mathrm{a}}$ & $0.55 \pm 0.02$ & $7.2 \pm 1.0$ \\
\hline Alloxan-diabetic (standard) & $0.70 \pm 0.06$ & $19 \pm 0.7^{\mathrm{a}}$ & $0.82 \pm 0.05$ & $33 \pm 5.7^{\mathrm{a}}$ & $0.42 \pm 0.02^{\mathrm{a}}$ & $19 \pm 3.8^{a}$ \\
\hline
\end{tabular}

Values are mean \pm SEM for not less than six animals [23]. ${ }^{a} p<0.01$ for difference from normal rats fed standard diet

Table 2. Activities of activator protein in rat tissues: effect of diet and diabetes

\begin{tabular}{lrr}
\hline Rat (diet) & \multicolumn{2}{l}{$\begin{array}{l}\text { Activator protein } \\
\text { (\% of control) in: }\end{array}$} \\
\cline { 2 - 3 } & \multicolumn{1}{c}{ Liver } & Kidney \\
\hline Normal (standard) & $100 \pm 14$ & $100 \pm 21$ \\
Normal (80\% casein) & $98 \pm 12$ & $173 \pm 27$ \\
Normal (9\% casein) & $10 \pm 1^{\mathrm{a}}$ & $30 \pm 5^{\mathrm{a}}$ \\
Normal (0\% casein) & $9 \pm 1^{\mathrm{a}}$ & $14 \pm 4^{\mathrm{a}}$ \\
Normal (48 h starved) & $28 \pm 4^{\mathrm{a}}$ & $21 \pm 4^{\mathrm{a}}$ \\
Diabetic (standard) & $30 \pm 2^{\mathrm{a}}$ & $155 \pm 26$ \\
\hline
\end{tabular}

Results are mean \pm SEM for not less than six animals [23].

${ }^{a} \mathrm{p}<0.01$ for difference from control (normal rats fet standard diet)

dria but not at all in liver mitochondria under normal conditions of incubation. In extracts of muscle mitochondria, inactivation by phosphorylation with ATP is rapid and essentially complete; in extracts of liver and kidney mitochondria, inactivation by phosphorylation is slower and incomplete. Upon purification, inactivation of liver and kidney BCKDH complex by phosphorylation is rapid and complete. These results suggested that in liver and kidney, but not muscle mitochondria, phosphorylation of BCKDH complex is inhibited by some factor which is lost during purification of the complex. This conclusion was supported by the finding that in tissues of normal rats in vivo approximately $90-95 \%$ of complex is in the inactive (phosphorylated) form in muscles whereas in liver and kidney only approximately $50 \%$ of complex is in the inactive form. As a working hypothesis it is presumed that the presence of activator protein (free E1) in liver and kidney prevents inactivation of the complex by phosphorylation. This hypothesis is capable of being put to the test when specific antibodies for activator protein are available to us.

\section{Effects of diet and of diabetes}

Methods have been developed which permit assay of activator protein, total complex, and percentage active form in rat tissues (without interference in liver and kidney by activator protein) [23, 24]. Some representative data from our studies are shown in Tables 1 and 2. It may be computed that in normal rats fed a standard diet at least $70 \%$ of active complex is hepatic (it may be greater because of reactivation of phosphorylated complex by activator protein, the extent of which cannot be assessed). In liver and kidney $55-70 \%$ of complex was in the active form; in muscles the percentage active complex was $5 \%$. Low protein diets or $48 \mathrm{~h}$ of starvation decreased total complex in liver but not in kidney or muscles; decreased percentage active complex in liver and kidney but not heart; and decreased activity of activator protein in liver and kidney. Overall low protein diets decreased the activity of branched chain complex by $90 \%$ in liver and by $70 \%$ in kidney with no obvious change in muscles. In affected tissues the mechanism involves loss of E1 activity (presumably by repression of synthesis, loss of total holocomplex activity, and accelerated inactivation by phosphorylation.

Untreated alloxan diabetes decreased the percentage active complex in liver and kidney, but increased it in heart; it decreased total complex in heart but not in liver or kidney; and it decreased activator protein in liver but not in kidney. Overall whole body active BCKDH complex was computed to be decreased by approximately $50 \%$ by untreated alloxan-diabetes. If comparable changes occur in uncontrolled human diabetes they could (in conjunction with tissue protein breakdown) provide an explanation for the increased circulating concentration of branched chain amino-acids.

\section{General discussion and conclusions}

Regulation of the PDH complex is of fundamental importance to animals including man. The stores of glycogen in animals are limited and suffice only to support blood glucose for approximately 12-24h. Adaptation to starvation requires suppression of glucose oxidation and substitution of fatty acids and/or ketone bodies as respiratory fuels. In prolonged starvation glucose oxidation may be almost totally suppressed; the possible exceptions being some cells in the central nervous system which may not be capable of oxidising ketone bodies. The low rate of glucose oxidation is sustained by gluconeogenesis from amino-acids and glycerol. The mechanisms which lead to inactivation of the PDH complex and which have been summarised here provide the basis for suppression of glucose oxidation in 
starvation. They are presumably initiated by deficient action of insulin and it is presumably insulin insufficiency which may result inappropriately in suppression of glucose oxidation in diabetes. The mechanisms are complex and involve lipolysis and lipolytic hormones and activation of PDH kinase by procedure of $\beta$-oxidation of fatty acids. They involve also longer term effects on specific protein synthesis of regulatory proteins possibly mediated by lipid fuels and hormones such as glucagon.

Regulation of the BCKDH complex is also known to be of fundamental importance. Clinical and biochemical observations would indicate that normal functioning of this complex is essential for the maintenence of normal circulating concentrations of branched chain amino- and ketoacids, normal brain development and life. Regulation of the BCKDH complex provides a mechanism which adjusts the rate of degradation of branched chain amino-acids in relation to dietary supply. The changes in activity which occur in starvation and in diabetes presumably suffice to allow for a modest increase in the circulating concentration of leucine. This may maintain basal insulin secretion and by this action and by direct action on tissue protein synthesis and degradation leucine may limit tissue protein degradation. A major role of reversible phosphorylation in the BCKDH complex in conjunction with the action of activator protein is assumed to result in preferential hepatic degradation of branched chain ketoacids. The reason for this is not entirely apparent but it may be related to the unique functions of the liver in respect of gluconeogenesis and ketogenesis for which products of branched chain ketoacid degradation are substrates.

Acknowledgements. I am grateful to the many students, postdoctoral fellows, and visiting fellows for their contribution to the work of my own laboratory over the past 20-25 years; and to the British Diabetic Association and the Medical Research Council (UK) for their support over this period.

\section{References}

1. Randle PJ (1966) Carbohydrate metabolism and lipid storage and breakdown in diabetes. Diabetologia 2: 237-247

2. Ashcroft SJH (1980) Glucoreceptor mechanisms and the control of insulin release and biosynthesis. Diabetologia 18: 5-15

3. Ashcroft FM, Harrison DE, Ashcroft SJH (1984) Glucose induces closure of single potassium channels in isolated rat pancreatic $\beta$ cells. Nature 312: 446-448

4. Struck E, Ashmore J, Wieland OH (1966) Effects of glucagon and long chain fatty acids on glucose production by isolated perfused rat liver. Adv Enzyme Regul 4: 219-224

5. Cuendet GS, Loten EG, Renold AE (1975) Evidence that the glucose fatty acid cycle is operative in isolated skeletal (soleus) muscle. Diabetologia 12: 336

6. Linn TC, Pettit FH, Reed LJ (1969) Regulation of the activity of pyruvate dehydrogenase complex by phosphorylation and dephosphorylation. Proc Natl Acad Sci USA 62: 234-241
7. Randle PJ (1983) Mitochondrial 2-oxoacid dehydrogenase complexes of animal tissues. Phil Trans R Soc Lond B 302: 47-57

8. Reed LJ (1981) Regulation of mammalian pyruvate dehydrogenase complex by a phosphorylation - dephosphorylation cycle. Curr Top Cell Regul 18: 95-106

9. Krzentowski G, Pirnay F, Pallikarakis N, Luyckx AS, Lacroix M, Mosora F, Lefebvre PJ (1981) Glucose utilization during exercise in normal and diabetic subjects. Diabetes 30:983-988

10. Randle PJ, Sugden PH, Kerbey AL, Radcliffe PM, Hutson NJ (1978) Regulation of pyruvate oxidation and the conservation of glucose. Biochem Soc Symp 43: 47-67

11. Denton RM, Brownsey RW, Belsham GJ (1981) A partial view of the mechanism of insulin action. Diabetologia 21: 347-362

12. Walser M, Williamson JR (eds) (1981) Metabolism and clinical implication of branched chain amino and ketoacids. Elsevier/ North Holland, New York Amsterdam Oxford

13. Randle PJ, Fatania HR, Lau KS (1984) Regulation of the mitochondrial branched chain 2-oxoacid dehydrogenase complex of animal tissues by reversible phosphorylation. In: Cohen P (ed) Enzyme regulation by reversible phosphorylation - further advances. Elsevier, Amsterdam New York Oxford, pp 1-26

14. Randle PJ (1981) Phosphorylation dephosphorylation cycles and the regulation of fuel selection in mammals. Curr Top Cell Regul 18: $107-128$

15. Wieland $\mathrm{OH}$, Siess EA, Weiss L, Löffler G, Patzelt C, Portenhauser R, Hartmann U, Schirman A (1973) Regulation of mammalian pyruvate dehydrogenase complex by covalent modification. Symp Soc Exp Biol 27: 371-400

16. Randle PJ, Fuller SJ, Kerbey AL, Sale GJ, Vary TC (1984) Molecular mechanisms regulating glucose oxidation in insulin deficient animals. In: Dumont JE, Nunez J (eds) Hormones and cell regulation, vol 8, INSERM European Symposium. Elsevier, Amsterdam New York Oxford, pp 139-150

17. Pearce FJ, Forster J, De Leeuw G, Williamson JR, Tutwiler GF (1979) Inhibition of fatty acid oxidation in normal and hypoxic perfused hearts by 2-tetradecylglycidic acid. J Mol Cell Cardiol 11: $893-915$

18. McCormack JG, Denton RM (1984) Role of $\mathrm{Ca}^{2+}$ ions in the regulation of intramitochondrial metabolism in rat heart. Biochem $J$ 218: $235-247$

19. Hassinen IE, Hultinen K (1975) Respiratory control in perfused rat heart. Biochem Biophys Acta 408:319-330

20. Kerbey AL, Richardson LJ, Randle PJ (1984) The roles of intrinsic kinase and of kinase/activator protein in the enhanced phosphorylation of pyruvate dehydrogenase complex in starvation. FEBS Lett 176: 115-119

21. Stansbie E (1976) Regulation of the human pyruvate dehydrogenase complex. Clin Sci Mol Med 51:445-452

22. Randle PJ, Patston PA, Espinal J (1985) Branched chain ketoacid dehydrogenase complex of animal tissues. In: Krebs EG, Boyer PD (eds) The enzymes, 3rd edn, Vol 18. Academic, Orlando (Fla) London (in press)

23. Espinal J, Patston P, Fatania HR, Lau KS, Randle PJ (1984) Purification and properties of a protein activator of phosphorylated branched chain 2-oxoacid dehydrogenase complex. Biochem $\mathbf{J}$ 225: 509-516

24. Patston PA, Espinal J, Randle PJ (1984) Effects of diet and of alloxan-diabetes on the activity of branched-chain 2-oxoacid dehydrogenase complex and of activator protein in rat tissues. Biochem J 222: 711-719

Professor P.J.Randle

Nuffield Department of Clinical Biochemistry

University of Oxford

John Radcliffe Hospital

Headington

Oxford OX3 9DV

England 